\section{A novel application of the adult Merocel nasal pack} Dear Sir,

Post-adenoidectomy haemorrhage is a potentially life-threatening complication with a prevalence of $0.38-1.5 \% .^{1-3}$ Post-nasal packs are the preferred haemostatic solution. Eighty-seven percent of ENT

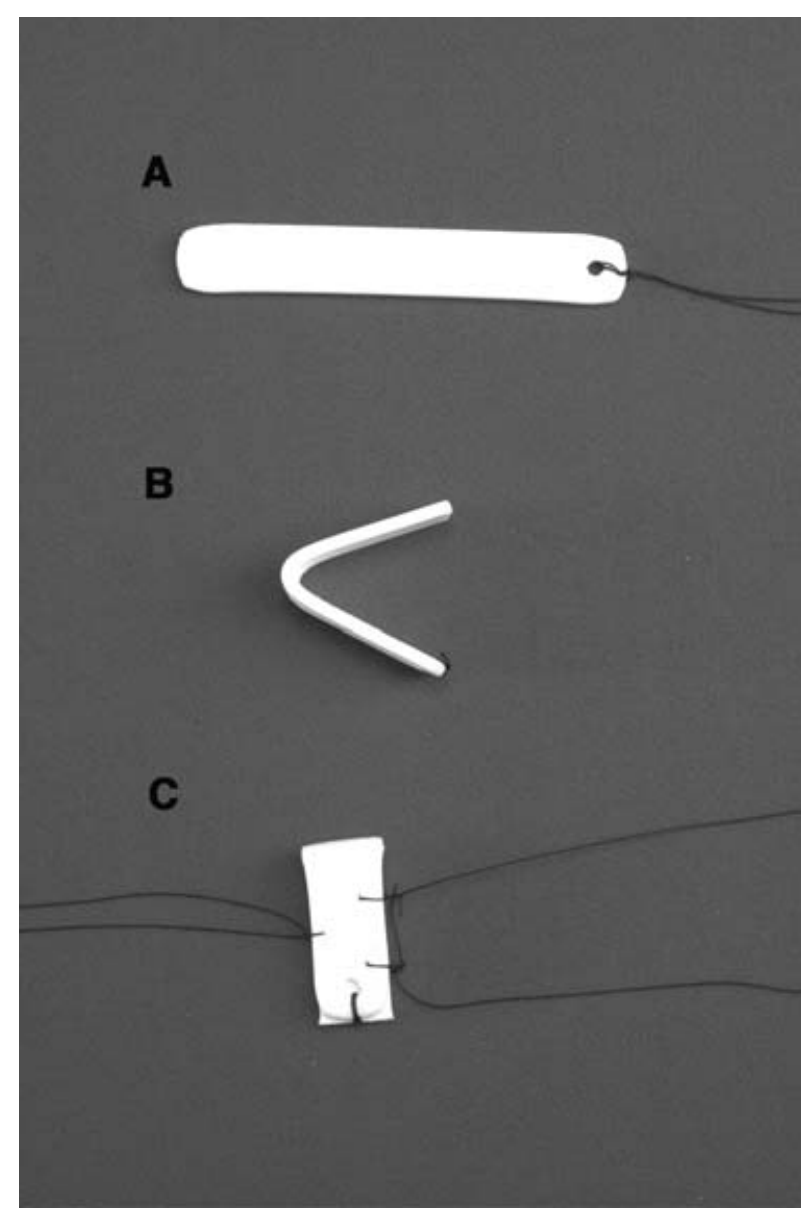

FIG. 1

A, an adult size $10 \mathrm{~cm}$ Merocel epistaxis pack; B, pack folded in half with ties removed; $C$, modified pack with sutures, ready for insertion. consultants questioned preferred using them, in a recent survey, with $78 \%$ leaving them in overnight. ${ }^{3}$

We recently treated a four-year-old girl with obstructive sleep apnoea. Unusually large adenoids were removed with an adenoid curettage, and six hours post-operatively the patient was found to have persistent haemorrhage from the post-nasal space. Sutures and diathermy failed to control the haemorrhage, so a pack was fashioned using an adult size $10 \mathrm{~cm}$ Merocel epistaxis pack. This was trimmed to size to fit transversely in the post-nasal space once, and the pack was folded in half and the pre-existing ties cut off (Figure 1b). Silk sutures were placed at the midpoint of the length of the pack (oral suture) and on the opposite side (nasal sutures) (Figure 1c) as for a conventional postnasal pack. Insertion was achieved by introducing Jaques's catheters via each nasal airway, and saline was applied to expand it. The pack was secured across a gauze bolster at the columella. After an uneventful night the pack was easily removed orally the following morning and the child was discharged.

This novel use of a modified standard adult Merocel pack was extremely well tolerated, effective and was easy to remove, and we hope that others might find this useful in similar circumstances.

V J Possamai, MRCS, H Griffiths, FRCS ORL-HNS, Department of Otolaryngology, Birmingham Heartlands Hospital, Birmingham, UK.

\section{References}

1 Ahmed K, McCormick MS, Baruah AK. Day-case adenoidectomy - is it safe? Clin Otolaryngol 1993;18:406-9

2 Guida RA, Mattucci KF. Tonsillectomy and adenoidectomy: an inpatient or outpatient procedure? Laryngoscope 1990; 100:491-3

3 Tzifa KT, Skinner DW. A survey on the management of reactionary haemorrhage following adenoidectomy in the UK and our practice. Clin Otolaryngol 2004;29:153-6 